\title{
Dynamic Multicontrast X-Ray Imaging Method Applied to Additive Manufacturing
}

\author{
Lorenzo Massimi $\odot,{ }^{1, *}$ Samuel J. Clark $\odot,{ }^{2, \dagger}$ Sebastian Marussi, ${ }^{2}$ Adam Doherty, ${ }^{1}$ Joachim Schulz $\odot,{ }^{3,4}$ \\ Shashidhara Marathe, ${ }^{5}$ Christoph Rau, ${ }^{5}$ Marco Endrizzi, ${ }^{1}$ Peter D. Lee $\odot,{ }^{2}$ and Alessandro Olivo ${ }^{1}$ \\ ${ }^{1}$ Department of Medical Physics and Biomedical Engineering, University College London, \\ Gower St, London WC1E 6BT, United Kingdom \\ ${ }^{2}$ Department of Mechanical Engineering, University College London, Gower St, London WC1E 6BT, United Kingdom \\ ${ }^{3}$ MicroWorks GmbH, Schnetzlerstrae 9, 76137 Karlsruhe, Germany \\ ${ }^{4}$ Institute of Microstructure Technology, Karlsruhe Institute of Technology, 76021 Karlsruhe, Germany \\ ${ }^{5}$ Diamond Light Source, Harwell Oxford Campus, OX11 ODE Didcot, United Kingdom
}

(Received 13 April 2021; revised 6 July 2021; accepted 12 October 2021; published 18 November 2021)

\begin{abstract}
We present a dynamic implementation of the beam-tracking x-ray imaging method providing absorption, phase, and ultrasmall angle scattering signals with microscopic resolution and high frame rate. We demonstrate the method's ability to capture dynamic processes with $22-\mathrm{ms}$ time resolution by investigating the melting of metals in laser additive manufacturing, which has so far been limited to single-modality synchrotron radiography. The simultaneous availability of three contrast channels enables earlier segmentation of droplets, tracking of powder dynamic, and estimation of unfused powder amounts, demonstrating that the method can provide additional information on melting processes.
\end{abstract}

DOI: 10.1103/PhysRevLett.127.215503

X-ray multicontrast imaging is based on the retrieval of phase changes and ultrasmall angle scattering (or dark field) occurring when $x$ rays pass through a sample, in addition to conventional attenuation [1-3], providing new, complementary information on the imaged samples. Phase imaging provides superior contrast in low-attenuating materials, which improves the visibility of small density changes [4-6]. Dark field highlights the presence of structures below the system's resolution [7,8]. Optical elements are used to retrieve these three imaging channels. Multiple frames are acquired while the optical element is displaced in different positions, which is at odds with the requirements of dynamic imaging. Although single-shot methods exist, they entail a reduction in resolution, either to the period of the optical element when gratings or masks are employed [9] or to the correlation window in specklebased methods [10]. Propagation-based methods are not quantitative in single-shot mode unless the sample is homogeneous [11]; only recently methods have emerged that provide some degree of access to dark field [12]. To overcome these limitations, we developed a method based on a dynamic implementation of the beam tracking (BT) approach [13]. As a single-shot method, BT's spatial resolution would also be limited to the optical elements (an absorption grating, usually referred to as mask) period. We addressed this by continuously translating the mask in front of the sample during the acquisition of an image sequence. The mask shapes the $\mathrm{x}$-ray beam into beamlets that are (dynamically) dampened, deflected, and broadened by the sample [see Fig. 1(a)], the analysis of which yields attenuation, differential phase, and dark-field images, respectively [13,14]. Laser additive manufacturing (LAM) enables the 3D printing of metallic components with previously unattainable structural complexity $[15,16]$ and has the potential to transform manufacturing. Dynamic $\mathrm{x}$-ray imaging has provided insights into the physics behind LAM by exploiting the high frame rate achievable at thirdgeneration synchrotron facilities [17-21]. However, these studies were limited to conventional attenuation and in-line phase contrast. Here, we present the first application of a multicontrast $\mathrm{x}$-ray dynamic imaging to the investigation of LAM processes. We show that the new contrast channels unlock additional insights into the melting and solidification process. This is obtained simultaneously and with a single setup, requiring minimum changes to existing in situ synchrotron LAM setups and acquisition pipelines.

The experiment was performed at the I13 beamline of the Diamond light source (United Kingdom), using a pink beam spectrum to maximize the flux [23]. The energy of the beam ranged approximately from 15 to $40 \mathrm{keV}$ with a maximum at about $21 \mathrm{keV}$. A schematic view of the setup is shown in Fig. 1(a). The beam is split into a series of beamlets by an absorption mask, producing $5 \mu \mathrm{m}$ wide beamlets, $20 \mu \mathrm{m}$ apart from each other. The relatively low duty cycle was chosen to keep the beamlets well separated, making their identification and analysis viable (see Supplemental Material [22], Fig. S1). To prevent spatial resolution from being limited by the mask period, we continuously moved the mask in front of the sample (see Supplemental Material [22] Video 1). In previous implementations, this was addressed by laterally scanning the sample in subperiod steps, referred to as "dithering" steps. 

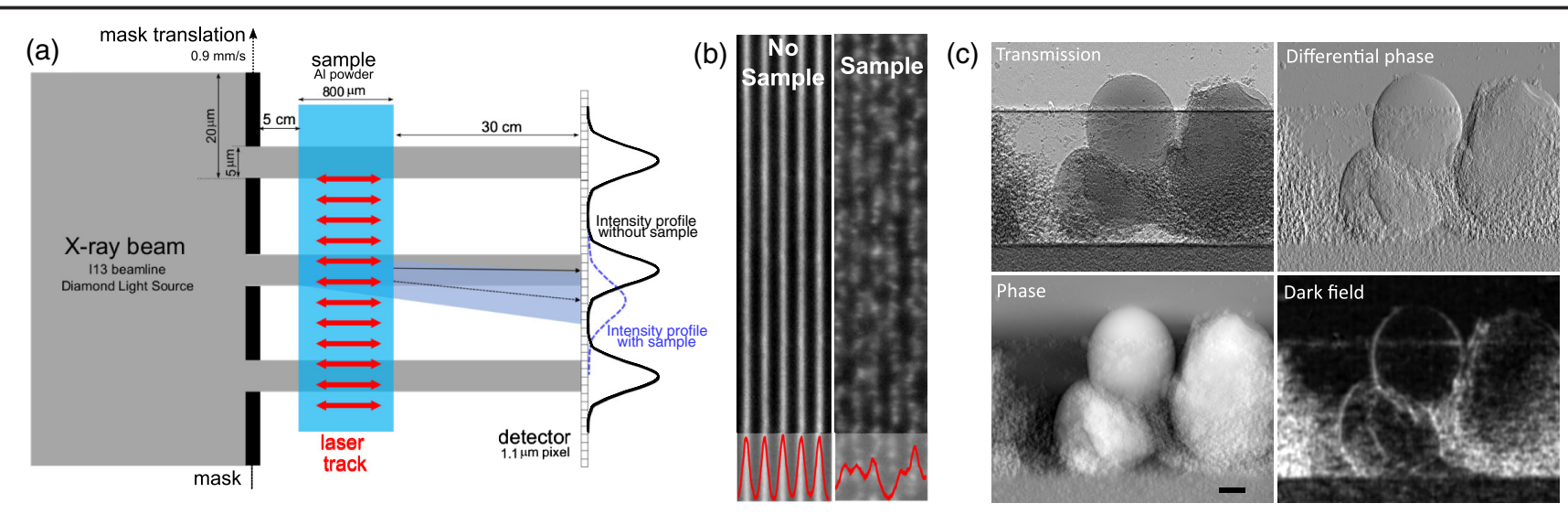

FIG. 1. (a) Schematic of the experimental setup. (b) The beamlets' shape with and without sample. (c) An example of the simultaneously obtained multicontrast images, including (clockwise from top left) transmission, differential phase, dark field, and phase. The scale bar is $200 \mu \mathrm{m}$. See Supplemental Material [22] Videos 2-5 for the time evolution of each contrast channel.

This leads to a resolution determined by the size of the apertures in the mask [24]. Although in this case we scanned the mask instead of the sample and used a continuous rather than a stepped movement, negligible differences are expected between this and the previously used dithered approach, so long as the mask is moved by exactly one aperture during each exposure. The reduce in resolution because of the continuous movement is given by the quadrature sum of mask aperture and amount by which the mask is moved during an exposure [25]. In this case they are both about $5 \mu \mathrm{m}$; hence a reduction from $5 \mu \mathrm{m}$ to $7 \mu \mathrm{m}$ is expected, with minimal impact on the images. Details are provided in the Supplemental Material [22], Figs. S2 and S3. The need to move the mask by one aperture during each exposure imposes a constraint on the mask speed, which must be equal to the ratio between mask aperture size and exposure time. This acquisition method affects time resolution because multiple images are combined to create the final one. In the present experiment, the limiting factor was the maximum speed of the mask scanning motor $(0.9 \mathrm{~mm} / \mathrm{s})$, which imposed an exposure time of $5.6 \mathrm{~ms}$ per image. Combined with an aperture size (and therefore a resolution) of $5 \mu \mathrm{m}$ and (the equivalent of) four dithering steps, this limited the time resolution to $22 \mathrm{~ms}$, which resulted in motion artefacts when fast changes occur in the sample (see Supplemental Material [22], Fig. S5). This can be improved in future experiments, as the use of faster linear translators $(100-1000 \mathrm{~mm} / \mathrm{s})$ would increase the time resolution by $2-3$ orders of magnitude, achieving a flux-limited exposure time. The mask was placed as close as possible to the sample, which was an $800 \mu \mathrm{m}$ thick $\mathrm{Al}$ [26] powder bed enclosed between two plates of glassy carbon and placed inside a sealed chamber fluxed with Ar [17,27]. Although a closer distance would have been beneficial, this chamber imposed a minimum mask-to-sample distance of $5 \mathrm{~cm}$. A laser was rastered above the surface of the powder bed [see Fig. 1(a)], simulating the hatch-pattern build of an overhang structure.
A power of $25 \mathrm{~W}$ was used. After each scan the sample was manually removed, and a second acquisition was performed with the same parameters to obtain the flat fields (see Supplemental Material [22] for details about data acquisition). This resulted in the formation of a beaded track as seen in Supplemental Material [22] Video 1. Despite the sharply cut apertures, the beamlets appear Gaussian shaped because of diffraction from the apertures, as visible in Fig. 1(b). With the sample in place, the beamlets' shape is drastically changed according to transmission, refraction, and scattering [see Fig. 1(b) and scheme in Fig. 1(a)]. Transmission changes the beamlets' intensity, while refraction displaces their position [14]. Powder creates large refraction angles, because of the multiple air-metal interfaces causing strong deformations of the beamlets' shape, as visible in Fig. 1(c). Small angle scattering changes the beamlets' width, which is difficult to assess visually [14]. The quantitative assessment of the changes in intensity, position, and width of each beamlet allows quantitative recovery of the transmission, differential phase, and small-angle scattering signals (respectively) from a single image [13]. This was performed by extracting a line profile for each detector row over five adjacent beamlets from images with and without the sample and fitting a 5-Gaussian profile to each (see Supplemental Material [22] and Ref. [28] for details). The differential phase image can be integrated, yielding a phase map of the sample [29]. Example images for each contrast channel are presented in Fig. 1(d), showing the droplets formed toward the end of the sequence (see Supplemental Material [22] Videos 2-5 for the dynamic evolution of each contrast channel). From Fig. 1(d), it is evident that phase exhibits a stronger droplet contrast against the remaining, unfused powder than transmission. Similarly, while a strong darkfield signal originates from the powder, a very low signal is observed within the formed droplet. Figure 1(d) and especially Supplemental Material [22] Videos 2-5 demonstrate that multicontrast imaging can be combined with 


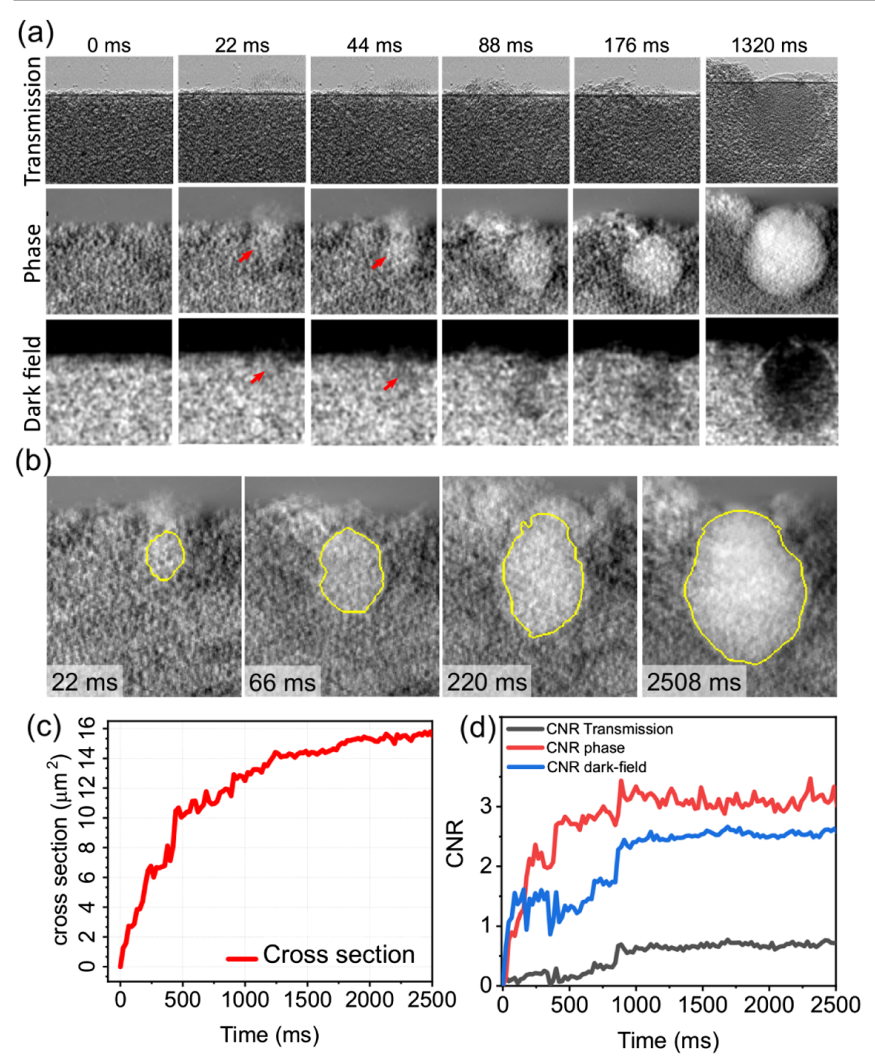

FIG. 2. (a) Image sequences (focused on one of the droplet formation areas) for the three contrast channels (top to bottom: transmission, phase, and dark-field), starting from the instant when the laser hits the powder. Red arrows point at changes in the powder, indicating the formation of the droplet in both phase and dark field. (b) The result of droplet segmentation using the phase signal. The graphs in (c) and (d) show the evolution of the cross section and contrast-to-noise ratio (CNR) of the droplet. The images have been blurred to have the same frequency content (see Supplemental Material [22], Fig. S4).

dynamic acquisitions, without the latter affecting the retrieval of the various contrast channels. Moreover, the results suggest that an aperture-driven resolution can be maintained in dynamic acquisitions, which offers the option to acquire multicontrast movies at a predetermined resolution level (see Supplemental Material [22], Fig. S2).

Phase and dark-field maps with improved contrast allow following the evolution of the droplet from very early stages, well before it becomes visible in transmission, as shown in Fig. 2. The sequences of Fig. 2(a) allow visually following the formation and evolution of a droplet, starting from the instant the laser hits the powder. After $22 \mathrm{~ms}$, a change in density becomes visible in the phase and dark field (see red arrows and Supplemental Material [22], Fig. S6), but not in transmission. The decrease in scatter intensity caused by the powder-air interfaces indicates that the powder is starting to melt. At the following time step (44 ms), changes in both phase and dark-field images are evident, while the droplet begins to become visible in transmission only around $176 \mathrm{~ms}$, when it has become totally apparent with very strong contrast, in both the other channels. The negligible dark-field signal within the droplet when it is fully formed $(t \sim 1300 \mathrm{~ms})$ indicates that the molten material has aggregated homogeneously for the beamlet size used. The improved sensitivity to density changes provided by phase-based methods allows using automatic segmentation [30] to quantitatively monitor the growth of the droplet from the earliest stages, long before this becomes possible with the attenuation-based images, despite these containing a significant contribution of freespace phase signal in our example (see Supplemental Material [22], Fig. S11). The result, based also on the assumption of a spheroidal shape of the droplet [31], is shown in Fig. 2(b). Following segmentation, the binary mask representing the droplet enables various quantitative assessments, for example, of how the cross section of the droplet evolves as a function of time [see Fig. 2(c)]. The same binary masks have been used to estimate the contrastto-noise ratio (CNR) of the droplet in the various image channels at different times [Fig. 2(d)]. This makes evident how the CNR for both phase and dark field is much higher than transmission at all the investigated times, confirming the previous qualitative observations about earlier detection and improved visibility (see Supplemental Material [22], Figs. S4, S7 and related discussion).

The strong differential signal prior to integration can be used to track the powder dynamic, by means of digital image correlation techniques. The results obtained using a tracking algorithm based on cross-correlation are shown in Fig. 3 (see the Supplemental Material [22], which includes Ref. [32], for details). The phase image, a map of the modulus of the velocity and of the flux are shown at two time steps, with the corresponding velocity vectorial field superimposed. Immediately after the laser hits the powder [see Fig. 3(a)], this is pushed downward with an average speed of $0.3 \mathrm{~mm} / \mathrm{s}$. An accumulation point is formed just beneath the surface, as shown in the flux map (red arrow), with the consequent formation of a depletion layer on the surface. At $t=160 \mathrm{~ms}$ [Fig. 3(b)], two accumulation points can be observed on top of the droplet (red arrows in the flux map). The powder is moving with a maximum speed of $0.44 \mathrm{~mm} / \mathrm{s}$ and converging toward a central region where almost no movement can be observed. This may indicate the formation of a molten layer on top of the droplet while the laser is moving in the direction of the x-ray beam. Indeed, after an additional $60 \mathrm{~ms}$, a feature with a density similar to that of the main droplet appears on top of it (yellow arrow in the inset of the phase image), indicating the formation of a new solidified volume, either connected to the droplet below or lying on top of it. These findings suggest that velocity, and especially flux maps, can enable identifying droplet formation areas before the droplets actually form. It is important to note that velocity and flux have been obtained 

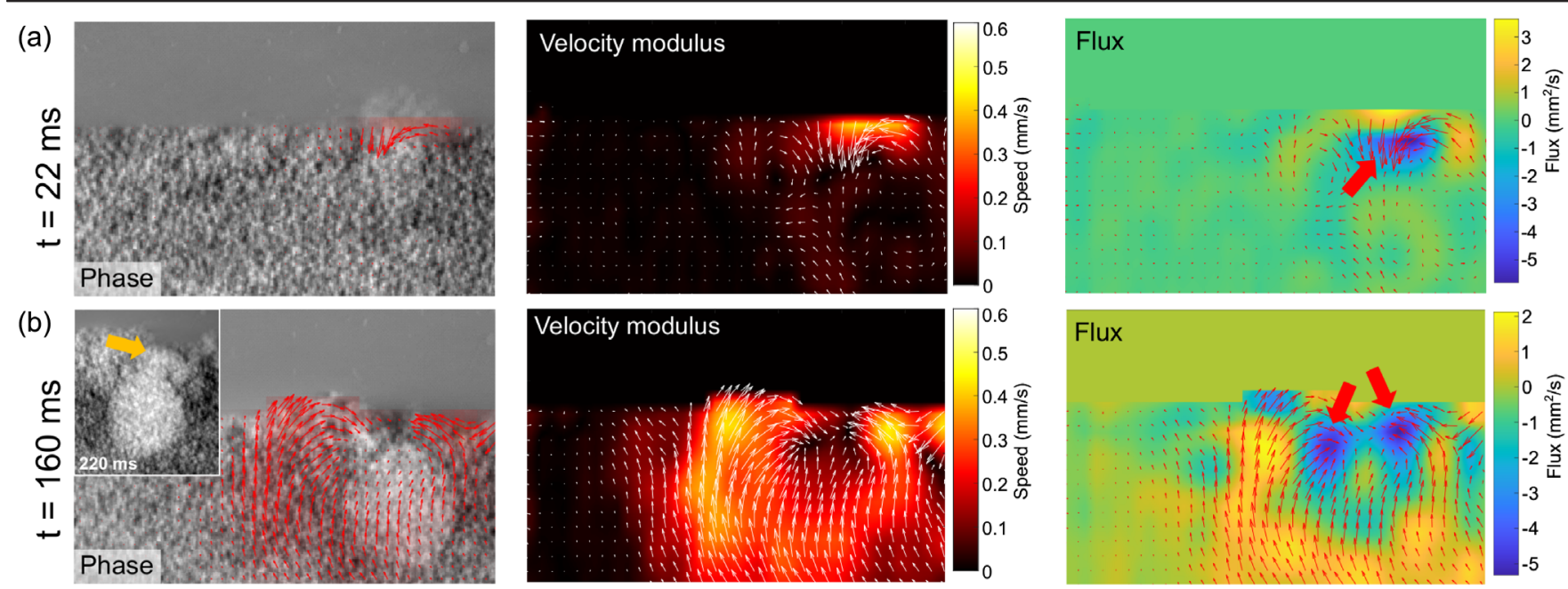

FIG. 3. (a), (b) Results from the powder tracking algorithm at different time steps: for each time step, the phase image with the velocity field overlapped, maps of the modulus of the velocity and of the flux are shown (left to right). The overlapped vectors are scaled differently between the phase and the velocity and flux images for aesthetic purposes. Note that velocity is projected onto the plane of the drawing, and the flux is integrated along the x-ray propagation direction. Times immediately after the laser hit the surface (a) and before the formation of a new molten track (b) have been chosen. The inset in the phase image in (b) shows a later time instant, after a droplet has completely formed.

from 2D radiographic projections. Therefore, the velocity field represents the projection of a $3 \mathrm{D}$ vector onto the plane orthogonal to x-ray beam direction, and the corresponding flux is integrated along the same line. This would become more limiting with increasing sample thickness; indeed, computed tomography (CT) acquisitions are needed to obtain full 3D information.

Although BT is compatible with CT, the footprint of the melting rig, alongside the high frame rate requirements needed to achieve high time resolution in $3 \mathrm{D}$, pose significant obstacles to the application of multicontrast CT to LAM. Here we propose a mechanism to extract some degree of 3D information along the x-ray propagation direction, providing an estimate of the amount of unfused powder along the sample thickness, at least at the proof-ofconcept level. If a single material is imaged, the intensity of the dark-field signal is linear with sample thickness [33]. Hence, the variation in dark-field intensity over time provides an estimate of the amount of unfused powder along the x-ray propagation direction, which can be made
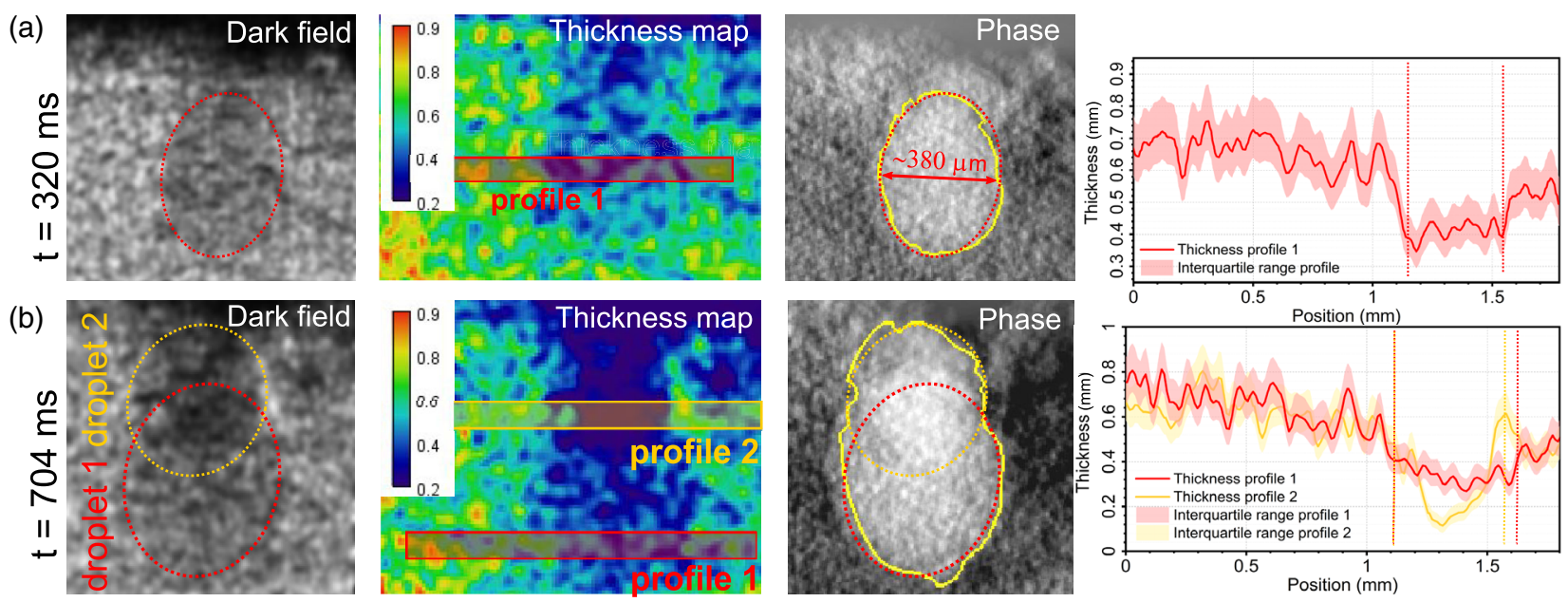

FIG. 4. (a),(b) For two time instants, dark-field and phase images alongside the corresponding powder thickness maps estimated from the dark-field signal intensity. Average line profiles across features of interest extracted from the thickness images are also reported for each panel with the corresponding interquartile prediction band. The shape of the droplet was obtained as the best ellipsoidal fit of the segmented perimeter. In (b), the perimeter was split in two parts to fit two ellipses. Vertical colored bars indicate the droplet size as determined from the fit. 
quantitative through calibration. Generally, the amount of powder on the beam path depends on grain orientation and packing, which are unknown. We estimated the initial thickness using Paganin's single-material phase retrieval [11] on a region imaged both with and without the mask. Representative images are reported in Fig. 4. The first time step in Fig. 4(a) $(t=320 \mathrm{~ms})$ shows how the formation of a droplet, clearly visible in the phase image, is accompanied by a decrease in the dark-field signal. The corresponding thickness map was obtained by inverting the linear relationship between scattering intensity and thickness of the powder bed, estimated independently, through Paganin's retrieval (see Supplemental Material [22], Fig. S13 and related discussion, which includes Ref. [34]). The thickness map and line profiles show that approximately (400 \pm 70) $\mu \mathrm{m}$ of unfused powder are still present in front of or behind the droplet. This is in agreement with the droplet's maximum diameter $(\sim 380 \mu \mathrm{m})$ estimated from the phase image: assuming a spheroidal shape for the droplet, the sum of the two numbers is compatible with the overall thickness of the powder bed of about $800 \mu \mathrm{m}$. It should be noted, however, that the relatively large grains used in this experiment (20-45 $\mu \mathrm{m}$; see Supplemental Material [22]) can contribute significantly to making profiles more irregular when a significant amount of unfused powder remains. At $t=704 \mathrm{~ms}$ [Fig. 4(b)] a second droplet is formed, partially overlapping the previous one, as visible in the phase image [note this is the same image previously shown in the inset of Fig. 3(b)]. Although the scattering signal highlights a difference between the two droplets (see arrows), likely due to the reduction in the amount of unfused powder, this is not appreciable in the phase image. Remarkably, the powder tracking analysis highlighted the formation of an accumulation point just above the first droplet before the new one formed (Supplemental Material [22], Fig. S12). The thickness map extracted from the darkfield signal reveals that more powder has melted to form the new droplet, while the previously formed one has not expanded. The line profiles show that the powder thickness across profile 1 remained unchanged (at about $400 \mu \mathrm{m}$ ), while it has reduced to about $(100 \pm 40) \mu \mathrm{m}$ across profile 2 , indicating the formation of a new droplet through the melting of additional powder. Because of the low-pass nature of the phase retrieval and some degree of uncertainty in the choice of the retrieval parameters, especially when a nonmonochromatic beam is used, this approach provides an error-prone thickness value on a local basis, which can be assumed to be true only on an average basis. However, the retrieved thickness still provides an indication of the amount of unfused powder along a path relative to the initial quantity. If sufficient powder grain size and packing are determined separately, for example, by micro-CT, then the 2D dark-field intensity could be calibrated against the real amount of powder in a given direction, and provide quantitatively reliable pixelwise estimates (see Supplemental Material [22], Fig. S14).

In summary, the proposed method can bring advantages in the analysis of LAM processes. The higher CNR of phase over conventional attenuation allows the visualization of droplet formation from its earliest stages. The strong signal provided by differential phase enables tracking the dynamics of the unfused powder through digital image correlation, which was shown to highlight droplet formation areas before the droplets actually form. The dark-field signal can be related to the average amount of powder traversed by $\mathrm{x}$ rays along their path, which allows extracting the projected thickness from a single 2D image. Following this demonstration in LAM, we expect the method to find additional uses across the life and physical sciences. BT is relatively easy to implement with higher energy $\mathrm{x}$ rays, necessary for the scaling up to thicker samples, and robust against decreased coherence conditions, making adaptations to laboratory sources more straightforward.

This project has received funding from the ATTRACT project funded by the EC under Grant Agreement No. 777222. Additional funding was received from EPSRC (Grants No. EP/T005408/1 and No. EP/ P006566/1). PDL (CiET1819/10) and AO (CiET1819/2/ 78) are supported by the Royal Academy of Engineering under their Chairs in Emerging Technologies scheme. M. E. is supported by the Royal Academy of Engineering under their Research Fellowships scheme. Laboratory space and facilities were provided by the Research Complex at Harwell. The authors gratefully acknowledge the provision of beamtime MG23760-1 on ID13-2 at the Diamond Light Source, and the assistance of the beamline scientists there.

*1.massimiphd@gmail.com

Present address: X-ray Science Division, Argonne National 344 Laboratory, 9700 S. Cass Avenue, Lemont, Illinois, USA.

[1] T. Davis, D. Gao, T. Gureyev, A. Stevenson, and S. Wilkins, Nature (London) 373, 595 (1995).

[2] S. Wilkins, T. E. Gureyev, D. Gao, A. Pogany, and A. Stevenson, Nature (London) 384, 335 (1996).

[3] F. Pfeiffer, T. Weitkamp, O. Bunk, and C. David, Nat. Phys. 2, 258 (2006).

[4] H. Hetterich, M. Willner, S. Fill, J. Herzen, F. Bamberg, A. Hipp, U. Schüller, S. Adam-Neumair, S. Wirth, M. Reiser et al., Radiology 271, 870 (2014).

[5] G. E. Barbone, A. Bravin, A. Mittone, S. Grosu, J. Ricke, G. Cavaletti, V. Djonov, and P. Coan, Radiology 298, 135 (2021).

[6] L. Massimi, I. Bukreeva, G. Santamaria, M. Fratini, A. Corbelli, F. Brun, S. Fumagalli, L. Maugeri, A. Pacureanu, P. Cloetens et al., NeuroImage 184, 490 (2019).

[7] A. Yaroshenko, F. G. Meinel, M. Bech, A. Tapfer, A. Velroyen, S. Schleede, S. Auweter, A. Bohla, A. O. Yildirim, K. Nikolaou et al., Radiology 269, 427 (2013).

[8] D. Shoukroun, L. Massimi, F. Iacoviello, M. Endrizzi, D. Bate, A. Olivo, and P. Fromme, Composites Part B 181, 107579 (2020). 
[9] E. Dreier, A. Bergamaschi, G. K. Kallon, R. Brönnimann, U. L. Olsen, A. Olivo, and M. Endrizzi, Appl. Phys. Lett. 117, 264101 (2020).

[10] M.-C. Zdora, J. Imaging 4, 60 (2018).

[11] D. Paganin, S. C. Mayo, T. E. Gureyev, P. R. Miller, and S. W. Wilkins, J. Microsc. 206, 33 (2002).

[12] T. Gureyev, D. Paganin, B. Arhatari, S. Taba, S. Lewis, P. Brennan, and H. Quiney, Phys. Med. Biol. 65, 215029 (2020).

[13] F. A. Vittoria, G. K. Kallon, D. Basta, P. C. Diemoz, I. K. Robinson, A. Olivo, and M. Endrizzi, Appl. Phys. Lett. 106, 224102 (2015).

[14] M. Endrizzi and A. Olivo, J. Phys. D 47, 505102 (2014).

[15] E. MacDonald and R. Wicker, Science 353, 1512 (2016).

[16] N. Jones, Nature (London) 487, 22 (2012).

[17] C. L. A. Leung, S. Marussi, R. C. Atwood, M. Towrie, P. J. Withers, and P. D. Lee, Nat. Commun. 9, 1355 (2018).

[18] C. Zhao, K. Fezzaa, R. W. Cunningham, H. Wen, F. De Carlo, L. Chen, A. D. Rollett, and T. Sun, Sci. Rep. 7, 1 (2017).

[19] S. M. H. Hojjatzadeh, N. D. Parab, W. Yan, Q. Guo, L. Xiong, C. Zhao, M. Qu, L. I. Escano, X. Xiao, K. Fezzaa et al., Nat. Commun. 10, 1 (2019).

[20] C. L. A. Leung, S. Marussi, M. Towrie, J. del Val Garcia, R. C. Atwood, A. J. Bodey, J. R. Jones, P. J. Withers, and P. D. Lee, Addit. Manufac. 24, 647 (2018).

[21] Y. Chen, S. J. Clark, C. L. A. Leung, L. Sinclair, S. Marussi, M. P. Olbinado, E. Boller, A. Rack, I. Todd, and P. D. Lee, Appl. Mater. Today 20, 100650 (2020).
[22] See Supplemental Material at http://link.aps.org/supplemental/ 10.1103/PhysRevLett.127.215503 for details about the experiment and analysis methodology.

[23] Z. Pešić, A. De Fanis, U. Wagner, and C. Rau, J. Phys. Conf. Ser. 425, 182003 (2013).

[24] P. C. Diemoz, F. A. Vittoria, and A. Olivo, Opt. Express 22, 15514 (2014).

[25] C. Hagen, P. Coan, A. Bravin, A. Olivo, and P. Diemoz, J. Appl. Phys. 118, 054901 (2015).

[26] P. Vora, R. Martinez, N. Hopkinson, I. Todd, and K. Mumtaz, Technologies 5, 24 (2017).

[27] L. Sinclair, C. L. A. Leung, S. Marussi, S. J. Clark, Y. Chen, M. P. Olbinado, A. Rack, J. Gardy, G. J. Baxter, and P. D. Lee, Addit. Manufac. 36, 101512 (2020).

[28] C. J. M. Jones, F. A. Vittoria, A. Olivo, M. Endrizzi, and P. R. Munro, Opt. Lett. 43, 3874 (2018).

[29] L. Massimi, I. Buchanan, A. Astolfo, M. Endrizzi, and A. Olivo, Opt. Express 28, 39677 (2020).

[30] I. Arganda-Carreras, V. Kaynig, C. Rueden, K. W. Eliceiri, J. Schindelin, A. Cardona, and H. Sebastian Seung, Bioinformatics 33, 2424 (2017).

[31] P. Lee and J. Hunt, Acta Mater. 45, 4155 (1997).

[32] D. Garcia, Exp. Fluids 50, 1247 (2011).

[33] M. Endrizzi, F. A. Vittoria, L. Rigon, D. Dreossi, F. Iacoviello, P. R. Shearing, and A. Olivo, Phys. Rev. Lett. 118, 243902 (2017).

[34] A. Likas, N. Vlassis, and J. J. Verbeek, Pattern Recognit. 36, 451 (2003). 